\title{
Anatomical characteristics of the lingual foramen in ancient skulls: a cone beam computed tomography study in an Anatolian population
}

\author{
K.O. Demiralp ${ }^{1}$, S. Bayrak², M. Orhan³ , A. Alan ${ }^{1}$, E.S. Kursun-Cakmak1, K. Orhan ${ }^{4}$ \\ ${ }^{1}$ Dentomaxillofacial Radiology Department, Ministry of Health, Ankara, Turkey \\ 2Dentomaxillofacial Radiology Department, Abant Izzet Baysal University, Dentistry Faculty, Bolu, Turkey \\ ${ }^{3}$ Dentomaxillofacial Radiology Department, Beykent University, Dentistry Faculty, Istanbul, Turkey \\ ${ }^{4}$ Dentomaxillofacial Radiology Department, Ankara University, Dentistry Faculty, Ankara, Turkey
}

[Received: 31 May 2017; Accepted: 29 September 2017]

Background: The purpose of this study is to evaluate the anatomical features of lingual foramina and their bony canals in Anatolian ancient mandibles (9-10 th century) by using cone beam computed tomography (CBCT).

Materials and methods: Fifty-eight ancient dry mandibles were scanned with CBCT. Lingual foramina were grouped into midline, paramedian, posterior foramina and combination of these groups. Midline group was also classified according to internal surface of the mandible (gonial tubercles [GTs]). The incidence, vertical distance and diameter of lingual foramina were measured according to age groups and gender.

Results: The incidence of the lingual foramen was 96.6\%. Midline of the symphysis had the highest incidence (34.4\%) of foramina ( $p<0.05)$, followed by both midline and paramedian type (32.8\%; $p<0.05)$. Classification in terms of GT represented class 3 as the most encountered group (28.6\%). Number of foramina observed in the mandibles ranged from 0 to 6 with the incidence of $3.4 \%$ and $32.8 \%$, respectively. The male and $<35$ years groups presented larger measurement values in midline region $(p<0.05)$.

Conclusions: Mandibular lingual foramina and bony canals are frequently present in ancient mandibles. When compared with modern subjects, similar findings are observed according to published literatures. CBCT is also proved to be an effective imaging modality in the detection of lingual foramina and canals in anthropological studies. (Folia Morphol 2018; 77, 3: 514-520)

Key words: cone beam computed tomography, oral anatomy, mandible neurovascularisation

\section{INTRODUCTION}

The lingual foramen is usually present in the midline on the lingual aspect of the anterior part of the mandible, at the level of superior or inferior to the gonial tubercles (GTs) which may have variations in number and position. It is typically visualised as a single round radiolucent canal with a well-defined opaque border $[5,18]$. Generally, multiple lingual foramina are observed in the inner part of the mandible. Despite taking part mostly in the midline region, lingual foramina can also

Address for correspondence: Dr. S. Bayrak, Abant Izzet Baysal University, Faculty of Dentisty, Dentomaxillofacial Radiology Department, Bolu, Turkey, tel: +90 374253 8361, fax: +90 374254 0066, e-mail: dtseval@hotmail.com 
be observed in paramedian and posterior regions. Most of these present with lingual bone canals which enters the body of the mandible transferring vascular and/or neural structures. Actually, blood vessels and nerves running through these lingual canals often generate anastomoses with neurovascular structures from the mandibular canal and its branches [2]. Studies on cadaver specimens and on radiological samples detected a high occurrence (97-100\%) of this entity. Its prevalence seemed to be correlated to aging [4].

The anterior mandibular region between the mental foramina, which was mostly considered as a safe surgical zone, must receive more attention in terms of the vital structures passing through the lingual foramen. Nerve injury and/or severe bleeding may occur if the nerves or arteries are injured. Several reports have indicated surgical complications such as the perforation of the lingual cortex or the injury of the sublingual and submental arteries, resulting in severe, life-threatening haemorrhage in the floor of the mouth [3].

It was shown that lingual foramina and their bony canal variations can clearly be visualised via cone beam computed tomography (CBCT). CBCT raised interest in the studies regarding anatomy of the structures of different species and among earlier modern humans. In the current literature the mandibular neurovascular bony structures are frequently documented in various species and ancient skulls in addition to living subjects [11]. Previous studies have handled mostly mandibular size and shape. Neurovascular bony structures have not been studied sufficiently up to now in ancient skulls in attempt to make a comparison with living subjects.

The objective of this study is to assess and compare the lingual foramen's anatomical characteristics of ancient Anatolian population (9-10 $0^{\text {th }}$ century) by using $\mathrm{CBCT}$.

\section{MATERIALS AND METHODS}

The study protocol was approved by the Ethics Committee of the Faculty of Dentistry, Abant izzet Baysal University, Bolu, Turkey (Number 2017/36; Date 25/5/2017). Fifty-eight ancient (9-10 th century) dry mandibles that were unearthed from certain archaeological excavations from Anatolia, Turkey, which dated approximately to the period of 800-1,000 A.C., were used. The sex and the approximate age of each skull were determined by two independent anthropologists through special cranium characteristics. In this study following parameters were considered when estimating the age for the adults: the symphyseal surfaces of the pubic and the auricular surfaces of the coxa, the aspect of the spongious tissue in humerus and femur, the closure of the cranial sutures and the tooth wear. In sexual diagnosis, the following parameters were considered: general shape of the pelvis, size of the greater sciatic notch, the shape of the sacrum, robustness of the skeleton, development of muscular joints and insertions, cranial relief, forehead shape, robustness and shape of the mandible. Fiftyeight dry mandibles from 9-10 $0^{\text {th }}$ century were derived from Ankara University, Faculty of Languages, History and Geography and Department of Anthropology. These samples were scanned with Planmeca Promax 3D Max CBCT (Planmeca Oy, Helsinki, Finland).

\section{Imaging of mandibles}

All dry mandibles were scanned by using a standard mandibular positioning. The exposure parameter settings included: $96 \mathrm{kVp}, 12 \mathrm{~mA}$. The exposure time was $18 \mathrm{~s}$ and $a 360^{\circ}$ turn was selected. After scanning, all constructions and measurements were performed on a 21.3-inch flat-panel colour-active matrix TFT medical display (NEC MultiSync MD215MG, Munchen, Germany) with a resolution of $2048 \times 2560$ at $75 \mathrm{~Hz}$ and $0.17-\mathrm{mm}$ dot pitch operated at 11.9 bits. All examinations were made by an experienced maxillofacial radiologist. The examiner was also permitted to use enhancements and orientation tools such as magnification, brightness, and contrast to improve visualisation. All axial, sagittal and coronal images were carefully examined under standardised viewing conditions.

The incidence and the location of the lingual foramen were assessed. Maximum width of the lingual foramen (horizontal/vertical), vertical distance of the lingual foramen to the lower border of mandible and alveolar crest of the mandible were measured (Fig. 1).

Lingual foramina were classified according to their location into following subgroups:

1. Midline lingual foramen (in the centre of symphysis);

2. Paramedian lingual foramen (from central incisors mesial to canines distal);

3. Posterior lingual foramen (from first premolar mesial to third molars distal);

4. Midline + paramedian;

5. Midline + posterior;

6. Paramedian + posterior;

7. Midline + paramedian + posterior (Fig. 2). 


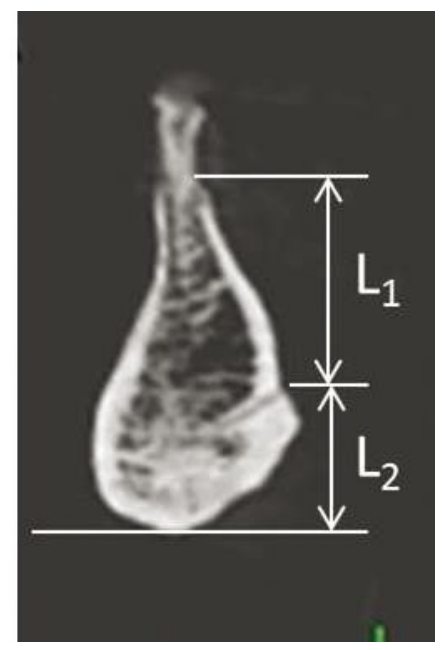

Figure 1. Measurement sites related to the lingual foramen on cone beam computed tomography sagittal image; $L_{1}$ - the distance between the lingual foramen and the alveolar crest; $\mathrm{L}_{2}$ - the distance between the lingual foramen and lower border of the mandible.

Finally, in the midline region, lingual foramina were classified into six subgroups according to location in terms of GT.

\section{Statistical analysis}

SPSS 17.0.1 (SPSS, Chicago, ILL) software programme was used to carry out the statistical analysis. In order to determine intra-observer variability, the observer performed the analysis twice with an interval of 2 weeks. To assess intra-observer reliability, the Wilcoxon matched-pairs signed rank test was used for repeat measurements. Mann-Whitney test and Kruskal-Wallis tests were used to compare measurements in terms of age groups and gender. Three age groups were separated: $18-35,35-55$ and $>55$ years. A p-value of less than or equal to 0.05 was considered statistically significant.

\section{RESULTS}

This study includes 58 mandibles belonging to $29(50 \%)$ female and $29(50 \%)$ male subjects. Eighteen (31\%) subjects were under 35 years, 21 (36.2\%) were between 35 and 55 years and 19 (32.8\%) were above 55 years.

The lingual foramen was detected with the incidence of $96.6 \%$ in 58 mandibles. In two mandibles, the lingual foramen was absent (3.4\%), while the rest of mandibles had the lingual foramen. The number of foramina which was recorded according the incidence was found as $32.8 \%(n=1), 17.2 \%$ $(n=7), 15.6(n=7), 12 \%(n=2), 10.3 \%(n=6), 8.7 \%$ $(n=5)$ (Table 1). All of the single lingual foramina were in the centre of symphysis. The mean horizontal and vertical maximum widths were detected as $0.86 \mathrm{~mm}$ and 0.93 , respectively.

The highest rate of the lingual foramen type was midline with the ratio $34.5 \%$ and followed by both midline and paramedian type (32.8\%) with statistical significant difference $(p<0.05)$. None of the paramedian and posterior types was seen individually (Table 2).

In midline area, lingual foramina and canals showed six different classes on the internal surface of the mandible (Fig. 3, Table 3). Class 3 (28.6\%) was the most common variation, followed by class 1 and 5 $(21.4 \%)$, class $4(14.3 \%)$, class $2(12.5 \%)$ and class 6 $(1.8 \%)$. The numbers of foramina found in this area varied from one to four with the following distribution, 1 foramen in $33.9 \%$, 2 foramina in $42.9 \%$, 3 foramina in $21.4 \%$ and 4 in $1.8 \%$.

In midline type, $62.1 \%$ had the diameter $\leq 1 \mathrm{~mm}$ (median $0.72 \mathrm{~mm}$, range $0.4-0.8 \mathrm{~mm}$ ) and $37.9 \%$ was $>1 \mathrm{~mm}$ (median $1.33 \mathrm{~mm}$, range $1.06-2.1 \mathrm{~mm}$ ). In the paramedian type, $65.3 \%$ of the lingual foramina had diameters $\leq 1 \mathrm{~mm}$ (median $0.77 \mathrm{~mm}$, range $0.4-1 \mathrm{~mm}$ ) and $34.7 \%$ were $>1 \mathrm{~mm}$ (median $1.23 \mathrm{~mm}$, range $1.04-1.43 \mathrm{~mm})$. In the posterior type,

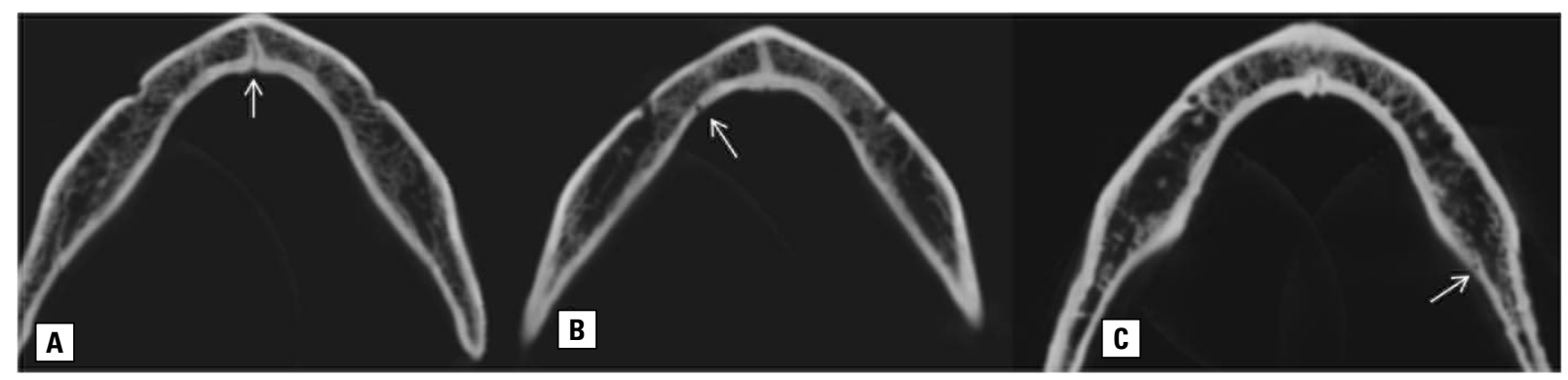

Figure 2. Axial view at the lingual foramen level showing midline type (A), paramedian type (B), and posterior type (C). 
Table 1. Numbers and frequencies of lingual foramen observed in ancient mandibles

\begin{tabular}{lc}
\hline Number & $\mathbf{N}(\%)$ \\
\hline None/non-visible & $2(3.4 \%)$ \\
One & $6(10.3 \%)$ \\
Two & $19(32.8 \%)$ \\
Three & $10(17.2 \%)$ \\
Four & $7(12.0 \%)$ \\
Five & $9(15.6 \%)$ \\
Six & $5(8.7 \%)$ \\
Total & $58(100 \%)$ \\
\hline
\end{tabular}

Table 2. Numbers, frequencies of anatomical classifications and quantity range of lingual foramina per anatomical classification

\begin{tabular}{lccc}
\hline Anatomical classification & N & $\%$ & Range \\
\hline None & 2 & 3.4 & - \\
Midline type & 20 & $34.5^{*}$ & $1-4$ \\
Paramedian type & 0 & 0 & - \\
Posterior type & 0 & 0 & - \\
Midline + paramedian type & 19 & $32.8^{*}$ & $1-5$ \\
Midline + posterior type & 9 & 15.5 & $1-5$ \\
Paramedian + posterior type & 0 & 0 & 0 \\
All types & 8 & 13.8 & $3-6$ \\
\hline
\end{tabular}

${ }^{*}$ Significant differences between groups
$66.7 \%$ of lingual foramina had diameters $\leq 1 \mathrm{~mm}$ (median $0.60 \mathrm{~mm}$, range $0.4-0.84 \mathrm{~mm}$ ) and $33.3 \%$ were $>1 \mathrm{~mm}$ (median $1.50 \mathrm{~mm}$, range $1.32-2.15 \mathrm{~mm}$ ).

In midline type the mean vertical distance from the superior border of the mandible to the lingual foramina was $11.00 \mathrm{~mm}$, from the inferior border of the mandible to the lingual foramina was $10.42 \mathrm{~mm}$. Significant difference was found in midline in terms of all measurements and existence of the lingual foramen. Male skulls showed larger values for all measurements than females. Moreover, group 1 ( $<35$ years) showed significant higher measurements than the other age groups $(p<0.05)$. Male skulls had more lingual foramina than female group in midline area $(p>0.05)$.

In the paramedian type the mean vertical distance from the superior border of the mandible to the lingual foramina was $8.17 \mathrm{~mm}$, the inferior border of the mandible to the lingual foramina was $9.93 \mathrm{~mm}$. No gender- and age-related differences in these distances were found $(p \geq 0.05)$.

In the posterior type the mean vertical distance from the superior border of the mandible to the lingual foramina was $7.41 \mathrm{~mm}$, from the inferior border of the mandible to the lingual foramina was $6.22 \mathrm{~mm}$. Neither sex nor age differences were found in the diameters studies $(p \geq 0.05)$.

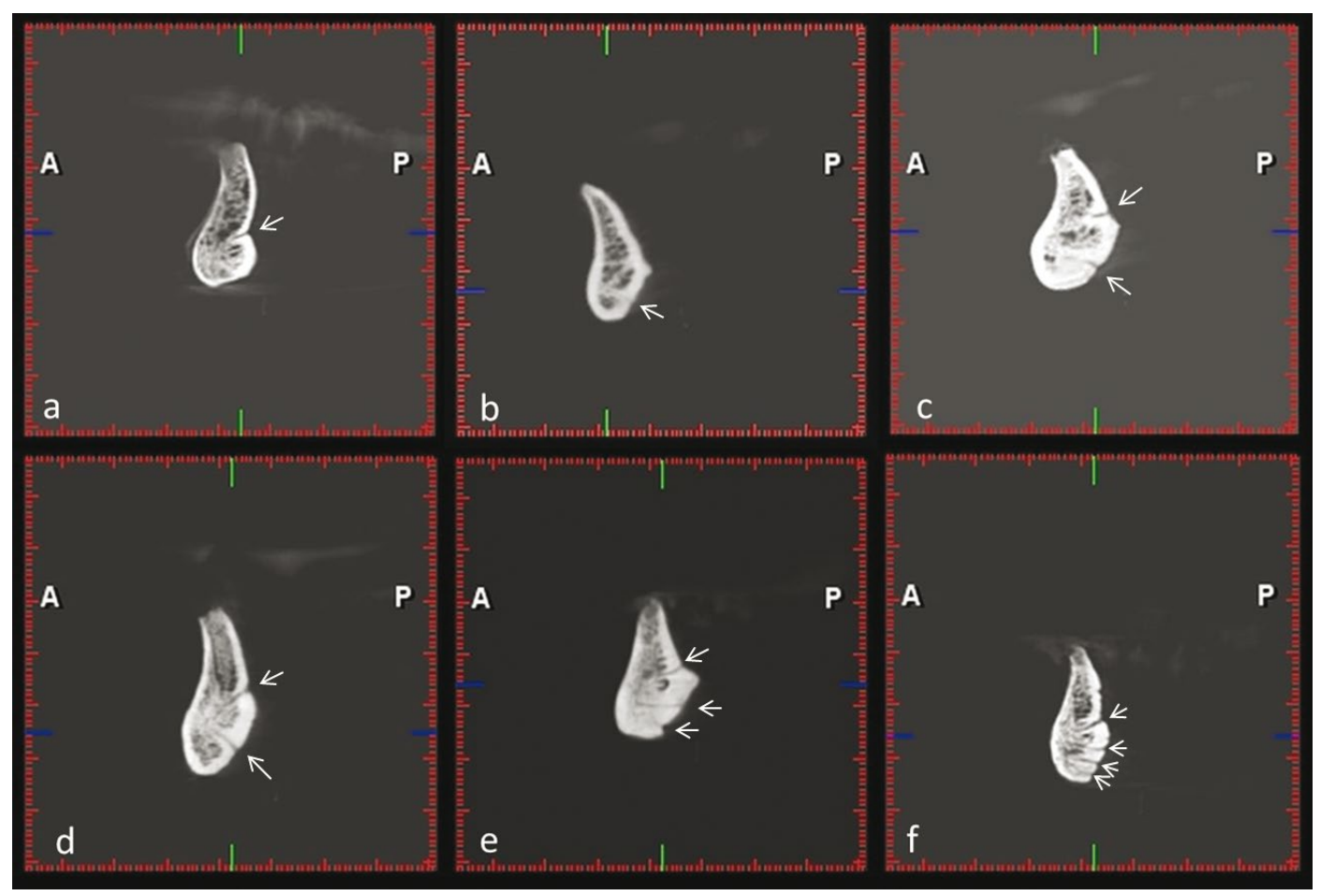

Figure 3. a-f. I-VI. Variations of the canals and foramina canals in the midline area of the mandible as observed during the review. 
Table 3. Description, variation and frequency of classes in midline area

\begin{tabular}{lc}
\hline Description of classification & $\mathbf{N}(\%)$ \\
\hline Class 1. Single lingual vascular canal located above the GT & $12(21.4 \%)$ \\
Class 2. Single lingual vascular canal located below the GT & $7(12.5 \%)$ \\
Class 3. Two canals (one located above, the other located below the GT) & $16(28.6 \%)$ \\
Class 4. Two canals (intersection of two separate canals; one oriented downwards — located above the GT, & $8(14.3 \%)$ \\
$\quad$ the other oriented upwards — located below the GT) & $12(21.4 \%)$ \\
Class 5. Three canals (one oriented downwards - located above the GT, others oriented upwards — located below the GT) & $1(1.8 \%)$ \\
\hline
\end{tabular}

GT — gonial tubercle

Table 4. Reported incidence and mean diameters of lingual foramen by various authors

\begin{tabular}{lcccc}
\hline Author's name [reference] & Year of publication & Study type & Incidence (\%) & Mean diameters \\
\hline Gahleither et al. [6] & 2001 & Radiological & 100 & $0.7 \pm 0.3$ \\
Tepper et al. [19] & 2001 & Radiological & 100 & $0.8 \pm 0.3$ \\
Vandewalle et al. [20] & 2006 & Cadaver/radiological & 98 & $0.6 \pm 0.2$ \\
Kawai et al. [10] & 2007 & Cadaver/radiological & 97 & - \\
Babiuc et al. [3] & 2011 & Radiological & 100 & $0.86 \pm 0.3$ \\
Sheikhi et al. [18] & 2012 & Radiological & 100 & $1.12 \pm 0.31$ \\
Bernardi et al. [4] & 2014 & Radiological & 77 & $0.9 \pm 0.25$ \\
Sekerci et al. [17] & 2014 & Radiological & 95.2 & $0.89 \pm 0.32$ \\
\hline
\end{tabular}

\section{DISCUSSION}

For tracing the origins of the human populations, mandible is indicated to be a better diagnostic tool rather than the cranium [12]. In identification of the fossil hominid remains, mandible descriptions have been used for comparative context. These approaches may have been driven by the fact that the linear measurements of the mandible have been shown to exhibit more variation than the crania and this greater variability has been related to mechanical loading $[8,12,14]$. Morphological variations of mandible among and between human species are linked to adaptations to demands on the masticatory systems [8].

Liang et al. [11] realised a comparative study on Neolithic, Medieval and 19-20 century skulls and found that highest mean values observed in 19-20 century samples for the mandibular and lingual canal in association with the highest lingual canal diameter and length strengthen the hypothesis that neurovascularisation has been taking place over time. However, these authors declared that the highest number of extra lingual canals is observed in Medieval group by refuting the foregoing argument.

To date, several reports have identified and measured lingual foramina in different ethnic groups by using anatomical dissection method [6, 15], traditional radiological method like orthopantomography and others $[9,16]$. However, no studies have been conducted to analyse, define and compare the anatomical characteristics of mandibular lingual foramina in the ancient Anatolian population. Some publications concerning the lingual foramen are given in (Table 4). Our results regarding the lingual foramen incidence $(96.6 \%)$ is compatible with the findings of previous reports.

Multiple no-name accessory foramina are observed in varied positions in the mandible especially in the midline region of lingual side. Liang et al. [13] and Katakami et al. [9] reported that the maximum number of the midline lingual foramina was four, while Sekerci et al. [17] indicated the number as three. Our findings showed parallel results in ancient mandibles: the highest number of midline lingual foramen was found four. It has been proposed that $\mathrm{CBCT}$ provides highly accurate data of the mandibular anatomy. Another reason for the difference in frequency noted may be anatomical variations in human mandibles from different geographical regions.

Tepper et al. [19] conducted a computed tomography study and reported that when there was only one foramen, it was frequently positioned superior to the GT. This finding is in accordance with the findings 
of Liang et al. [12] and Sekerci et al. [17] and our results. In the current study, single lingual foramen was localised superior and inferior to GT with the ratio of $21.4 \%$ and $12.5 \%$, respectively.

The location of the midline lingual foramina is important to avoid bleeding complications. The present study showed a mean distance of $10.42 \mathrm{~mm}$ from the lower mandibular border to the midline lingual foramina. This mean distance has been confirmed in many other studies: $10.0 \mathrm{~mm}$ [19], $10.2 \mathrm{~mm} \mathrm{[6],}$ $10.6 \mathrm{~mm}$ [12], $9.34 \mathrm{~mm}$ [17].

Yildirim et al. [21] and $\mathrm{He}$ at al. [7] estimated that $75.6 \%$ and $78.77 \%$ of lingual foramen, respectively, had diameters $\leq 1 \mathrm{~mm}$. In this study, the ratios of lingual foramina $\leq 1 \mathrm{~mm}$ are $62.1 \%, 65.3 \%$ and $66.7 \%$ for midline, paramedian and posterior types, respectively.

Tepper et al. [19] found the anterior lingual foramen in all of the examined patients $(n=70)$. Of these, $43 \%$ presented 1 canal, $27 \%$ had 2 canals, and $8.6 \%$ showed 3 canals. Aoun et al. [1] found no statistically significant differences between male and female group for neither the number of lingual canals nor the position of the foramina. They estimated most of the lingual foramina (76.64\%) were located above the GT, and the remaining were located below the tubercles. In the current study, male group had more lingual foramina and larger measurement values than female group in midline area.

Babiuc et al. [3] classified the lingual canals in relation to GT and stated that the canals were found right above them in $63.3 \%$ of the cases, below in $13.34 \%$ and both above and below (when there were multiple canals) in $23.3 \%$ of the cases. These results are incompatible with our findings; the most frequently seen type was one canal located above GT and the other canal located below GT (28.6\%).

\section{CONCLUSIONS}

In conclusion, the results of this study showed that mandibular lingual foramina and bony canals are frequently present in ancient mandibles. In the centre of symphysis, the incidence of foramina was the highest and in this region male and $<35$ years groups presented larger measurement values. Also there have been minor discrepancies in studied populations' measurements according to locations, age and gender; no significant variation was observed between the current literature results. There have been no studies in the professional literature to com- pare contemporary human mandibles with ancient mandibles.

\section{REFERENCES}

1. Aoun G, Nasseh I, Sokhn S, et al. Lingual foramina and canals of the mandible: anatomic variations in a lebanese population. J Clin Imaging Sci. 2017; 7: 16, doi: 10.4103/ jcis.JCIS_15_17, indexed in Pubmed: 28589055.

2. Arx von T, Lozanoff S. Lingual foramina and canals. Clinical Oral Anat. 2016: 463-487, doi: 10.1007/978-3319-41993-0.

3. Babiuc I, Tărlungeanu I, Păuna M. Cone beam computed tomography observations of the lingual foramina and their bony canals in the median region of the mandible. Rom J Morphol Embryol. 2011; 52(3): 827-829, indexed in Pubmed: 21892525.

4. Bernardi S, Bianchi S, Continenza MA, et al. Frequency and anatomical features of the mandibular lingual foramina: systematic review and meta-analysis. Surg Radiol Anat. 2017; 39(12): 1349-1357, doi: 10.1007/s00276-0171888-x, indexed in Pubmed: 28616681.

5. Denny C, Natarajan S, Ahmed J, et al. Anatomic Variation in Lingual Foramen: A Cone beam Computed Tomography Study. World J Dent. 2016; 7(4): 179-181, doi: 10.5005/ jp-journals-10015-1391.

6. Gahleitner A, Hofschneider U, Tepper G, et al. Lingual vascular canals of the mandible: evaluation with dental CT. Radiology. 2001; 220(1): 186-189, doi: 10.1148/radiology.220.1.r01jl05186, indexed in Pubmed: 11425994.

7. He X, Jiang J, Cai W, et al. Assessment of the appearance, location and morphology of mandibular lingual foramina using cone beam computed tomography. Int Dent J. 2016; 66(5): 272-279, doi: 10.1111/idj.12242, indexed in Pubmed: 27177988.

8. Humphrey LT, Dean MC, Stringer CB. Morphological variation in great ape and modern human mandibles. J Anat. 1999; 195 (Pt 4): 491-513, indexed in Pubmed: 10634689.

9. Katakami K, Mishima A, Kuribayashi A, et al. Anatomical characteristics of the mandibular lingual foramina observed on limited cone-beam CT images. Clin Oral Implants Res. 2009; 20(4): 386-390, doi: 10.1111/j.16000501.2008.01632.x, indexed in Pubmed: 19298292.

10. Kawai T, Asaumi R, Sato I, et al. Classification of the lingual foramina and their bony canals in the median region of the mandible: cone beam computed tomography observations of dry Japanese mandibles. Oral Radiol. 2007; 23(2): 42-48, doi: 10.1007/s11282-007-0064-0.

11. Liang $X$, Jacobs R, Corpas LS, et al. Chronologic and geographic variability of neurovascular structures in the human mandible. Forensic Sci Int. 2009; 190(1-3): 24-32, doi: 10.1016/j.forsciint.2009.05.006, indexed in Pubmed: 19525074.

12. Liang $X$, Jacobs $R$, Lambrichts $I$, et al. Microanatomical and histological assessment of the content of superior genial spinal foramen and its bony canal. Dentomaxillofac Radiol. 2005; 34(6): 362-368, doi: 10.1259/dmfr/75895125, indexed in Pubmed: 16227480.

13. Liang $X$, Jacobs $R$, Lambrichts I. An assessment on spiral CT scan of the superior and inferior genial spinal foramina and canals. Surg Radiol Anat. 2006; 28(1): 
98-104, doi: 10.1007/s00276-005-0055-y, indexed in Pubmed: 16341824.

14. Lycett SJ, Collard M. Do homoiologies impede phylogenetic analyses of the fossil hominids? An assessment based on extant papionin craniodental morphology. J Hum Evol. 2005; 49(5): 618-642, doi: 10.1016/j.jhevol.2005.07.004, indexed in Pubmed: 16125752

15. McDonnell D, Reza Nouri M, Todd ME. The mandibular lingual foramen: a consistent arterial foramen in the middle of the mandible. J Anat. 1994; 184 ( Pt 2): 363-369, indexed in Pubmed: 8014127.

16. Patil S, Matsuda Y, Okano T. Accessory mandibular foramina: a CT study of 300 cases. Surg Radiol Anat. 2013; 35(4): 323-330, doi: 10.1007/s00276-012-1044-6, indexed in Pubmed: 23192239.

17. Sekerci $A E$, Sisman Y, Payveren MA. Evaluation of location and dimensions of mandibular lingual foramina using cone-beam computed tomography. Surg Radiol Anat. 2014; 36(9): 857-864, doi: 10.1007/s00276-014-1311-9, indexed in Pubmed: 24831961.
18. Sheikhi M, Mosavat F, Ahmadi A. Assessing the anatomical variations of lingual foramen and its bony canals with CBCT taken from 102 patients in Isfahan. Dent Res J (Isfahan). 2012; 9(Suppl 1): S45-S51, indexed in Pubmed: 23814561.

19. Tepper G, Hofschneider UB, Gahleitner A, et al. Computed tomographic diagnosis and localization of bone canals in the mandibular interforaminal region for prevention of bleeding complications during implant surgery. Int J Oral Maxillofac Implants. 2001; 16(1): 68-72, indexed in Pubmed: 11280364.

20. Vandewalle G, Liang $X$, Jacobs $R$, et al. Macroanatomic and radiologic characteristics of the superior genial spinal foramen and its bony canal. Int J Oral Maxillofac Implants. 2006; 21(4): 581-586, indexed in Pubmed: 16955609.

21. Yildirim YD, Güncü GN, Galindo-Moreno P, et al. Evaluation of mandibular lingual foramina related to dental implant treatment with computerized tomography: a multicenter clinical study. Implant Dent. 2014; 23(1): 57-63, doi: 10.1097/ ID.0000000000000012, indexed in Pubmed: 24394340. 